\title{
Systematic review and meta-analysis of the effect of nutritional support on the clinical outcome of patients with traumatic brain injury
}

\author{
Lu Yang ${ }^{1}$, Dengbin Liao ${ }^{1}$, Xiaoling Hou ${ }^{1}$, You Wang ${ }^{1}$, Chaohua Yang ${ }^{2}$ \\ ${ }^{1}$ Department of Orthopedic Surgery, West China Hospital, Sichuan University/West China School of Nursing, Sichuan University, Chengdu, China; \\ ${ }^{2}$ Department of Neurosurgery, West China Hospital, Sichuan University/West China School of Nursing, Sichuan University, Chengdu, China \\ Contributions: (I) Conception and design: L Yang; (II) Administrative support: D Liao; (III) Provision of study materials or patients: X Hou; (IV) \\ Collection and assembly of data: Y Wang; (V) Data analysis and interpretation: C Yang; (VI) Manuscript writing: All authors; (VII) Final approval of \\ manuscript: All authors. \\ Correspondence to: Chaohua Yang. Department of Neurosurgery, West China Hospital, Sichuan University/West China School of Nursing, Sichuan \\ University, Chengdu 610041, China. Email: yangchaohua202109@163.com.
}

Backgrounda It remains unclear whether nutritional support can reduce the mortality and infection rate of
patients with traumatic brain injury (TBI), improve their gastrointestinal function, and shorten the length of
stay in the intensive care unit (ICU). The purpose of this study is to evaluate the effect of nutritional support
on the clinical outcome of TBI patients.
Methods: A computer search was conducted of the PubMed, Cochrane Library, Embase, Wanfang, and
China National Knowledge Infrastructure (CNKI) databases for randomized controlled trials investigating
the impact of nutritional support on the clinical outcomes of patients with TBI. The search included the
period from the establishment of the database to July 2021 . Two researchers independently screened the
literature, extracted the data, and evaluated the risk of bias in the included studies. RevMan 5.3 statistical
software (Cochrane Collaboration) was used to analyze the effect size, and a funnel plot was used to detect
publication bias.

Results: Seven articles reporting on 260 patients receiving nutritional support therapy compared with 252 standard nutrition control patients were included in the study. Meta-analysis showed that there was no significant difference in mortality between the nutritional support and standard nutrition treatments (RR $=0.74 ; 95 \%$ CI: $0.34-1.60 ; \mathrm{P}=0.44)$. However, there were significant differences in total serum protein levels $(\mathrm{MD}=2.23$; 95\% CI: 1.38-3.07; $\mathrm{P}<0.00001)$, total infection rates ( $\mathrm{RR}=0.54 ; 95 \% \mathrm{CI}: 0.41-0.71 ; \mathrm{P}<0.0001)$, lung infection rates $(\mathrm{RR}=0.60 ; 95 \% \mathrm{CI}: 0.45-0.81 ; \mathrm{P}=0.0006)$, length of stay in ICU ( $\mathrm{MD}=-5.65 ; 95 \% \mathrm{CI}$ : -6.18 to $-5.13 ; \mathrm{P}<0.00001)$ and Glasgow Coma Scale scores ( $\mathrm{MD}=2.77 ; 95 \% \mathrm{CI}: 1.75-3.78 ; \mathrm{P}<0.00001)$.

Discussion: Nutritional support effectively shortens the hospital stay of patients, reduces the infection rate of patients, and has a positive effect on promoting rehabilitation for patients with TBI. However, highquality, large-sample, multi-center randomized controlled trials are needed to further study the specific implementation standards of nutritional support.

Keywords: Traumatic brain injury (TBI); nutritional support; clinical outcome; meta-analysis

Submitted Sep 30, 2021. Accepted for publication Nov 18, 2021.

doi: 10.21037/apm-21-3071

View this article at: https://dx.doi.org/10.21037/apm-21-3071 


\section{Introduction}

Traumatic brain injury (TBI) is damage to the brain tissue caused by direct or indirect violence to the head. The Glasgow Coma Scale (GCS) defines severe TBI as a coma lasting more than 6 hours after injury. TBI is a common presentation in neurosurgery (1-3) and accounts for 13-21\% of all craniocerebral injuries. It is a complex and changeable life-threatening condition with a high fatality rate and poor prognosis that imposes a heavy burden on the patient's family and society $(1,4)$. Patients with TBI experience varying degrees of coma, cannot eat spontaneously, their body functions are affected by trauma and display clear signs of systemic metabolic stress. Traumatic brain injury frequently results in nutritional and metabolic disorders as the body's original nutritional reserves are consumed in large quantities, resulting in patients suffering from varying degrees of malnutrition soon after admission (1,5).

Parenteral nutrition $(\mathrm{PN})$ and enteral nutrition $(\mathrm{EN})$ are commonly used to maintain the nutritional status of patients with TBI. PN supplies nutrition to the patient via an intravenous route, whereas EN provides the necessary nutrients for metabolism through the gastrointestinal tract $(6,7)$. Active and reasonable nutritional support can improve overall treatment and prognosis. Given the complications associated with $\mathrm{PN}$ and gastrointestinal stress dysfunction, $\mathrm{EN}$ - especially early EN-has received increasing attention and has become the first choice for surgical nutritional support (5).

In March 2017, "Early enteral nutrition in critically ill patients: ESICM clinical practice guidelines" was published by the European Society of Intensive Care Medicine (ESICM) (5). The guidelines recommend that critically ill patients should be started on enteral nutrition within 24 hours of being admitted to the ICU. Therefore, the implementation of enteral nutrition during this time is defined as early enteral nutrition. Beyond 24 hours, it is regarded as late enteral nutrition. Early enteral nutrition is an important way to correct systemic metabolic disorders in TBI patients, improve immunity, and improve clinical outcomes $(8,9)$. However, $50-80 \%$ of TBI patients cannot tolerate enteral nutrition in the first 2 weeks after injury, need parenteral nutrition. Gastrointestinal dysfunction such as abdominal distension, diarrhea, and gastric retention may occur $(8,10)$. Implementing nutritional support can effectively improve the gastrointestinal flora, thereby protecting the gastrointestinal mucosal barrier and improving the body's immunity $(11,12)$.
The term "nutritional support" refers to the addition of supplementary nutrients to the standard nutritional support given to patients to enable them to achieve a higher level of comprehensive and valuable nutrition. However, whether early enteral nutrition combined with nutritional support can reduce fatality, infection, and gastrointestinal complication rates or improve immunity in TBI patients remains inconclusive. Additionally, previous studies have been limited by insufficient sample sizes and low statistical power, resulting in conflicting results. To this end, the present study used a meta-analysis to merge the results from existing studies to objectively evaluate the effect of nutritional support combined with early enteral nutrition on TBI patients so as to provide an evidence-based foundation for clinical practice in this field. We present the following article in accordance with the PRISMA reporting checklist (available at https://dx.doi.org/10.21037/apm-21-3071).

\section{Methods}

\section{Search strategy}

A computer search of the PubMed, Cochrane Library, Embase, Wanfang, and China National Knowledge Infrastructure (CNKI) databases located studies focused on enteral/parenteral nutrition, as well as guidelines and unpublished related literature. The search encompassed the period from the establishment of the databases to July 2021 and also included relevant manual literature searches. The search terms and strategy were as follows: \#1 “Craniocerebral Injury", \#2 “Traumatic Brain Injury", \#3 “Enhanced Nutrition", \#4 "Nutrition Support", \#5 "Probiotics", \#6 "Glutamine", \#7 "Randomized Controlled Trials", \#8 “Arginine”, and \#9 "Ly Acid”. We also combined \#1 and \#2 AND (\#3 OR \#4 OR \#5 OR \#6 OR \#7 OR \#8 OR \#9).

\section{Inclusion criteria}

To be included in the meta-analysis, studies were required to meet the following criteria: (I) the study design was a prospective randomized controlled trial (RCT); (II) the included participants were diagnosed with TBI; (III) the trial included at least one study group and a control group and provided measurable outcome indicators, such as nutritional indicators (e.g., total serum protein), outcome indicators (e.g., fatality rate), adverse reaction indicators (e.g., lung infection rate, total infection rate), Glasgow 
Coma Scale (GCS) scores, and length of stay in hospital. The most recent published articles were selected in instances where research data was duplicated.

\section{Exclusion criteria}

Studies were excluded according to the following criteria: (I) duplicate articles or those with insufficient data or of low quality; (II) studies that did not report on the observation indicators required for this study; (III) studies in which valid data could not be obtained or those which were expert opinions, summaries, or case reports; (IV) the research method was a non-randomized control (non-RCT); (V) studies that included patients with non-TBI or traumatic craniocerebral injury combined with gastrointestinal or abdominal injury.

\section{Literature screening and data extraction}

Two researchers independently screened the literature according to the inclusion and exclusion criteria and extracted the relevant information with a pre-designed data extraction table, including (I) basic characteristics of the study, including the name of the first author, publication year, country, and sample size; (II) characteristics of the research subjects and average GCS scores; (III) intervention-related information, including nutritional support type, nutrition method, nutrition start time, feeding route, feeding method, and duration of nutrition support; (IV) outcome indicators, including total protein levels, fatality rates, total infection rates, and lung infection rates. Researchers cross-checked the extracted information individually, and, if there were differences of opinion, they were resolved through discussion or consultation with a third party. If the information in the study was incomplete, the authors were contacted to obtain the relevant information; if the relevant data was not available, the article was excluded.

\section{Quality assessment}

A quality evaluation of the included studies was conducted using Cochrane's risk-of-bias assessment tool. The specific evaluation items were as follows: (I) whether the random allocation plan was correct; (II) whether the allocation was concealed; (III) whether blinding was used for the research subjects and researchers; (IV) whether blinding was used for the evaluation of the results; $(\mathrm{V})$ whether the outcome data were complete; (VI) whether there was selective reporting of the outcome; (VII) any other biases reported. Each included study was evaluated according to the above seven items, and an evaluation was made of "low risk," "unclear," and "high risk." A "low risk" assessment indicated a low risk of bias; an "unclear" assessment indicated there was insufficient relevant information to judge the level of risk or the bias was unclear, leading to a moderate risk of bias; a "high risk" assessment indicated a higher risk of bias. This work was independently evaluated by two researchers who had received statistical and related professional training and could independently complete quality evaluations. In the event of a disagreement over the evaluation conclusions, a third party was consulted for discussion and resolution.

\section{Statistical analysis}

The Revman 5.3 statistical software (Cochrane Collaboration) was used to merge the effect sizes. Categorical variables were analyzed using relative risk (RR). Continuous data were analyzed using the mean difference (MD) or standardized mean difference (SMD), and the $95 \%$ confidence interval (CI) of each effect size was calculated. The $\mathrm{I}^{2}$ test was used to test the heterogeneity between studies, and a sensitivity analysis was carried out according to the Cochrane systematic review method. If the heterogeneity between studies was small $\left(\mathrm{P}>0.1, \mathrm{I}^{2}<50 \%\right)$, a fixed-effects model was used to combine the effect sizes; when apparent heterogeneity was observed $\left(\mathrm{P} \leq 0.1, \mathrm{I}^{2} \geq 50 \%\right)$, a random-effects model was used to merge the effect size. A subgroup analysis was performed on the outcome indicators of the study. When five or more studies were available, a funnel plot was used to assess publication bias.

\section{Results}

\section{Search results and study characteristics}

According to the search strategy, a total of 906 related articles were retrieved, including 425 in the PubMed database, 304 in the Embase database, 43 in the Cochrane Library, 72 in the Wanfang database, and 62 in the CNKI database. After 266 duplicate articles were deleted, 640 articles for retrieval. Preliminary screening was conducted by reading the article abstracts, followed by a full-text reading of the 73 articles remaining after the initial screening. Finally, seven papers were included in this study, as shown in Figure 1. 

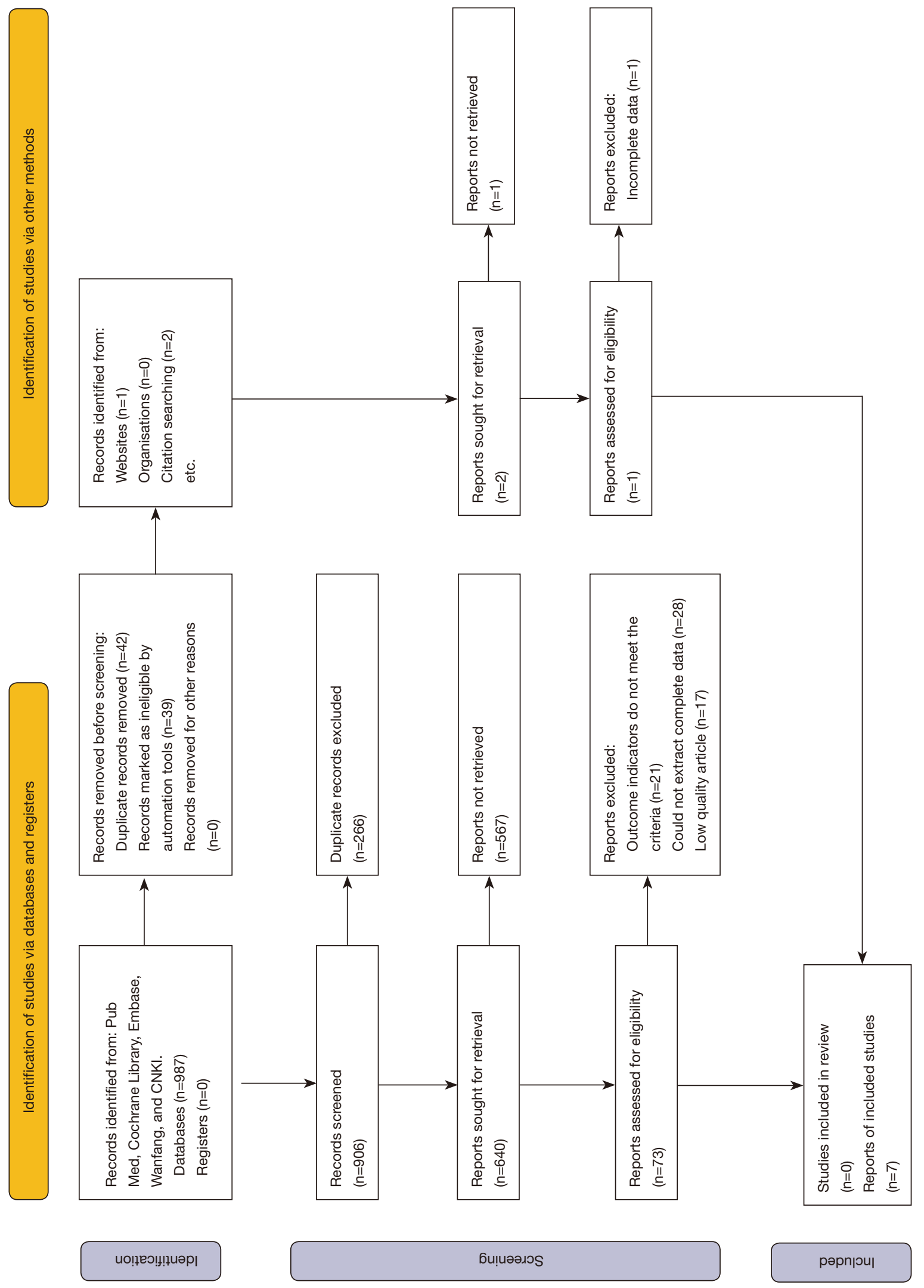

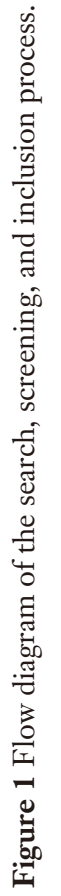


The seven included articles reported on 512 subjects in total and were published between 2011 and 2019. Four papers were in Chinese and three in English. The sample size included in a single study ranged from 36-114 cases. The basic characteristics of the studies are shown in Table 1 .

\section{Meta-analysis results}

\section{Total serum protein}

A total of two studies $(3,9)$ reported the effect of nutritional support intervention on the total protein level of patients with TBI. The results of the heterogeneity analysis indicated apparent homogeneity $\left(\mathrm{I}^{2}=0 \%, \mathrm{P}=0.69\right)$, so a fixed-effects model was used for the analysis. The combined effect size of the fixed effects model was $M D=2.23,95 \%$ CI (1.38-3.07), $\mathrm{P}<0.00001$. The results showed that the total serum protein level of patients in the nutritional support group was significantly higher than that of the standard nutrition group, as shown in Figure 2.

\section{Case fatality rate}

A total of four articles $(7,13,14,16)$ reported the related outcomes of nutritional support on the mortality of patients with TBI. The results of the heterogeneity test $\left(\mathrm{I}^{2}=53 \%\right.$; $\mathrm{P}=0.09$ ) indicated the presence of heterogeneity. The combined effect size of the random-effects model was $\mathrm{RR}=0.74,95 \% \mathrm{CI}(0.34-1.60), \mathrm{P}=0.44$, suggesting that, compared with standard nutrition, nutritional support did not significantly reduce the mortality of patients with TBI, as shown in Figure 3.

\section{Total infection rate}

Five articles $(7,9,14-16)$ reported the occurrence of total infections in patients with TBI after nutritional support intervention. The analysis indicated $\mathrm{I}^{2}=0 \%, \mathrm{P}=0.73$, and the combined analysis using a fixed-effects model showed that the total infection rate of the nutritional support group was lower than that of the standard nutrition group, $\mathrm{RR}=0.54$, 95\% CI (0.41-0.71), and the difference was statistically significant $(\mathrm{P}<0.0001)$, as shown in Figure 4.

\section{Lung infection rate}

Four articles $(7,13,14,16)$ reported the occurrence of lung infections in patients with TBI after nutritional support intervention. The analysis indicated $\mathrm{I}^{2}=0 \%, \mathrm{P}=0.99$, and the comprehensive analysis results of the fixed effects model showed that the lung infection rate of the nutritional support group was lower than that of the standard nutrition group, $\mathrm{RR}=0.60,95 \% \mathrm{CI}(0.45-0.81)$, and the difference was statistically significant $(\mathrm{P}=0.0006)$, as shown in Figure 5 .

\section{ICU hospital stay}

A total of four studies $(7,13,14,16)$ reported the impact of nutritional support intervention on the length of ICU stay in patients with TBI. The results of the heterogeneity analysis showed apparent homogeneity $\left(\mathrm{I}^{2}=0 \%, \mathrm{P}=0.41\right)$, so a fixed-effects model was used for the combined analysis. The combined effect size of the fixed effects model was $\mathrm{MD}=-5.65,95 \%$ CI $(-6.18$ to -5.13$), \mathrm{P}<0.00001$. The results showed that the length of ICU stay for patients in the nutritional support group was shorter than that of the standard nutrition group, and the difference was statistically significant, as shown in Figure 6.

\section{GCS score}

A total of three studies $(7,13,16)$ reported the effect of nutritional support intervention on the 15-day GCS score of patients with traumatic head injury. The result of the heterogeneity analysis indicated apparent heterogeneity $\left(\mathrm{I}^{2}=73 \% ; \mathrm{P}=0.02\right)$, so a random-effects model was used for the comparisons. The combined effect size of the random-effects model was MD $=2.77,95 \%$ CI (1.75-3.78), $\mathrm{P}<0.00001$. The results showed that the 15-day GCS score of patients in the nutritional support group was better than that of the standard nutrition group, and the difference was statistically significant, as shown in Figure 7.

\section{Publication bias}

Five articles reported on the total infection rate of patients with TBI, so a funnel plot was used to check the publication bias of the complete infection rate data. Visual inspection of the funnel plot showed asymmetry, indicating the possibility of publication bias in the total infection rate data (Figure 8). Publication bias analyses could not be performed on the reporting of total serum protein, mortality, pulmonary infection rate, ICU length of stay, or GCS scores as there were less than five studies that reported these indicators, which is below the publication bias requirements.

\section{Risk of bias}

All articles displayed a low risk of bias in random sequence generation. Six studies had a low risk of bias for allocation concealment $(3,9,13-16)$. The risk of bias in the blinding 
Table 1 Basic characteristics of the study articles

\begin{tabular}{|c|c|c|c|c|c|}
\hline Author & Country & Year & Journal & Intensive (n) & Control (n) \\
\hline Wan et al. (13) & China & 2020 & Clinical Research Report & 38 & 38 \\
\hline Tan et al. (14) & China & 2011 & Critical Care & 26 & 26 \\
\hline Yang et al. (15) & China & 2017 & Chin J Microecol & 43 & 43 \\
\hline Xiong et al. (16) & China & 2013 & $J$ Third Mil Med Univ & 17 & 21 \\
\hline Chen et al. (7) & China & 2015 & Chin J Nosocomiol & 57 & 53 \\
\hline
\end{tabular}

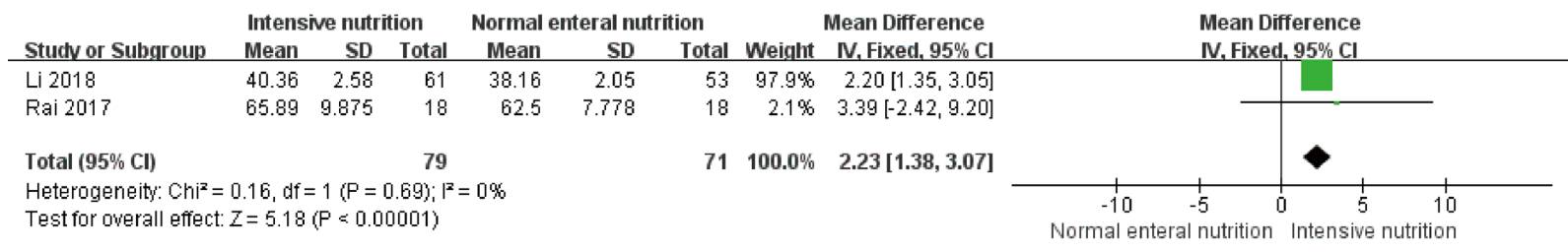

Figure 2 Forest plot of total serum protein. Comparison of total serum protein between the nutritional support group and the normal enteral group. Statistical method: inverse variance of fixed effects model [mean difference (MD) and 95\% confidence interval (CI)].

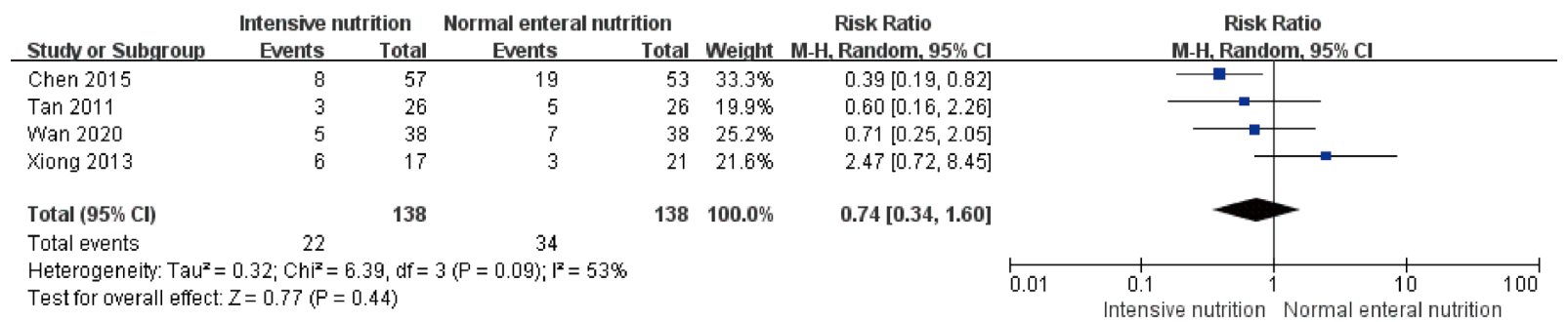

Figure 3 Forest plot of one-month case fatality rate. Comparison of one-month case fatality rate between the nutritional support group and the normal enteral group. Statistical method: Mantel-Haenszel of the random effects model [relative risk (RR) and $95 \%$ confidence interval $(\mathrm{CI})]$.

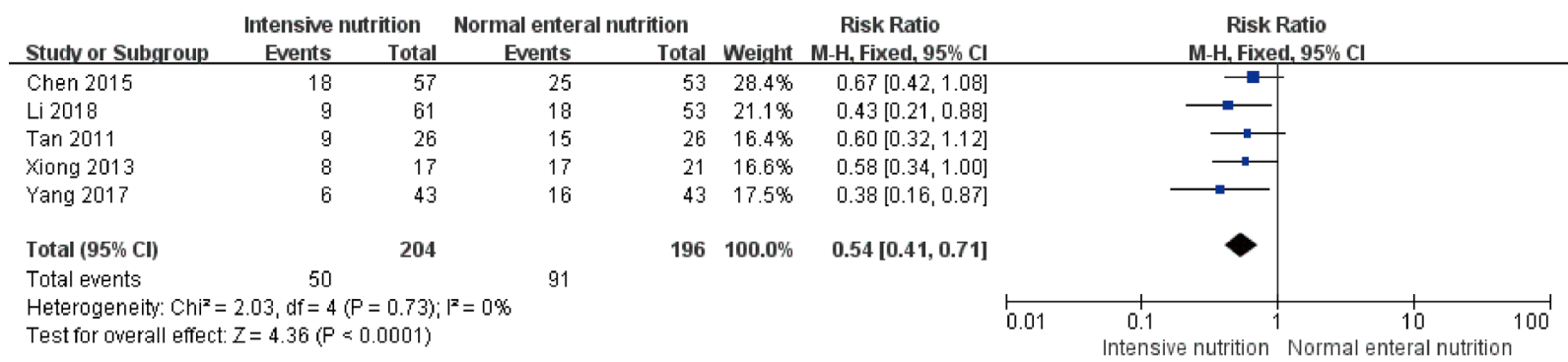

Figure 4 Forest plot of total infection rate. Comparison of total infection rate between the nutritional support group and the normal enteral group. Statistical method: Mantel-Haenszel of the fixed effects model [relative risk (RR) and $95 \%$ confidence interval $(\mathrm{CI})]$. 


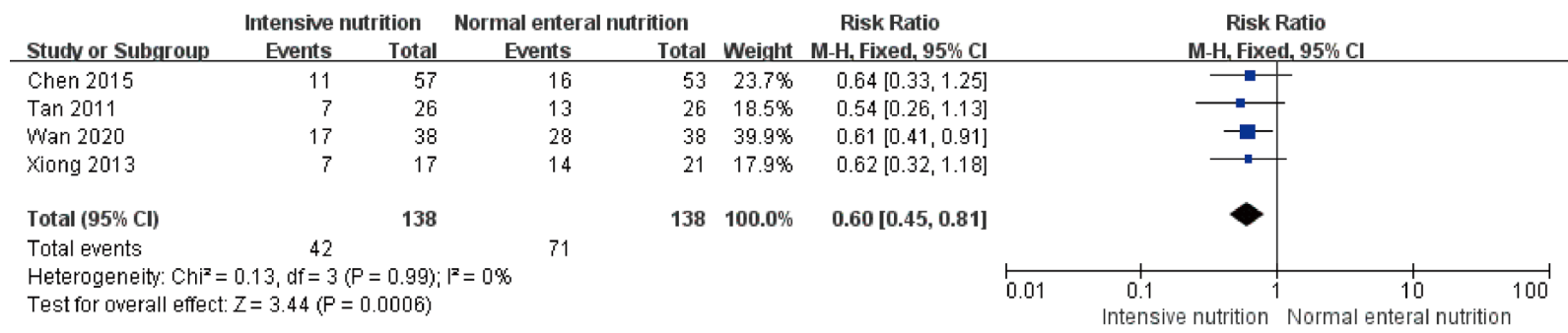

Figure 5 Forest plot of lung infection rate. Comparison of lung infection rate between the nutritional support group and the normal enteral group. Statistical method: Mantel-Haenszel of the fixed effects model [relative risk (RR) and 95\% confidence interval $(\mathrm{CI})$.

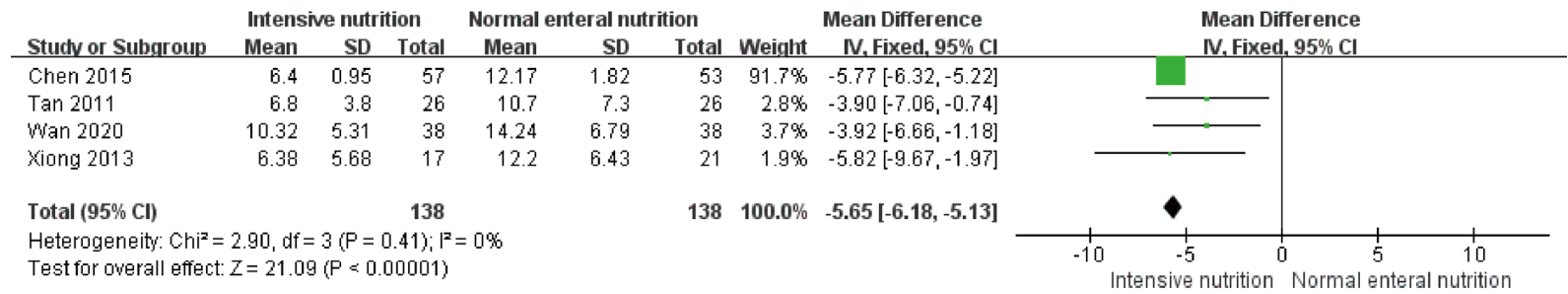

Figure 6 Forest plot of ICU hospital stay. Comparison of ICU hospital stay between the nutritional support group and the normal enteral group. Statistical method: inverse variance of the fixed effects model [mean difference (MD) and $95 \%$ confidence interval (CI)].

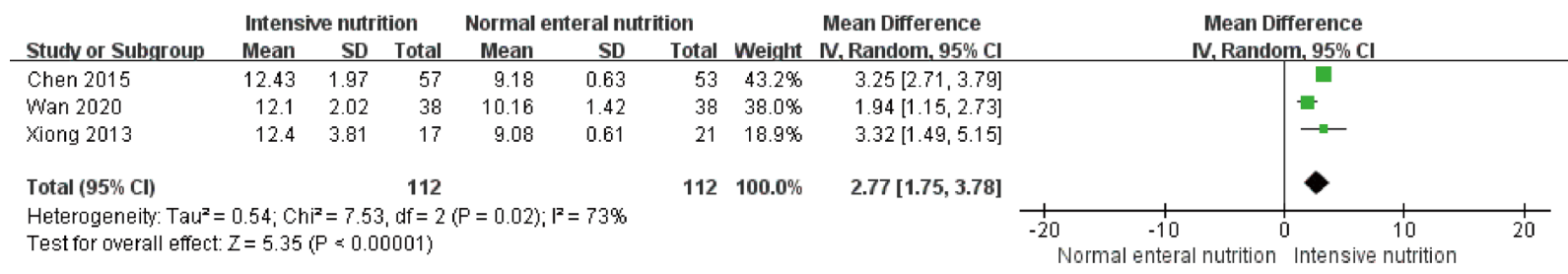

Figure 7 Forest plot of 15-day GCS score. Comparison of 15-day GCS score between the nutritional support group and the normal enteral group. Statistical method: inverse variance of the random effects model [mean difference (MD) and 95\% confidence interval (CI)].

of participants and researchers was low in four studies $(3,7,9,14)$ but high in three studies $(13,15,16)$. Five articles had a low risk of bias in the assessment of the blinded results $(7,9,13,14,16)$, whereas two studies had a high risk of bias $(3,15)$. All articles had a low risk of bias for incomplete outcome data, selective reporting domains, or other biases.

\section{Discussion}

The metabolism and decomposition rates in the body of a patient with TBI are 1.4 times faster than those of a normal person $(10,12)$. The body's systemic metabolism is disrupted, the body's energy consumption increases, protein decomposition accelerates, and the patient develops traumatic hypoproteinemia, which escalates the process of brain damage and increases the mortality rate (17). Therefore, timely and effective nutritional support is particularly important. Consciousness disorder caused by traumatic head injury makes it difficult for patients to eat, which can cause gastrointestinal and digestive dysfunction that leads to insufficient nutrient intake, thus hindering the body's ability to recover. Early enteral nutrition support for 


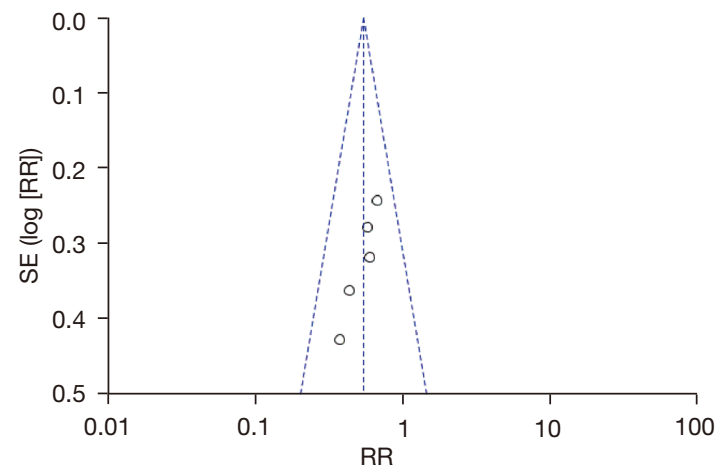

Figure 8 Total infection rate funnel plot. RR, relative risk; SE, standard error of the mean.

patients with TBI can improve immunity, promote wound healing, and the recovery of nerve function $(5,6)$. Intensified nutritional supplementation can be used to provide adequate dietary needs for patients with TBI. The use of nutritional support combined with early enteral nutrition in treating patients with TBI can effectively improve the nutritional status of the body after surgery, enhance nutritional support, and have a significant effect on the recovery of the patient's condition $(2,17,18)$.

Nutritional therapy is an integral part of the clinical treatment of patients with TBI and can promote postoperative recovery and reduce the occurrence of related complications $(19,20)$. In enteral/parenteral nutrition, adding glutamine, alanyl-glutamyl, probiotics, growth hormone, and other fortified nutritional preparations has a significant protective effect on the intestinal mucosal barrier function after stress (21-23), enteral nutrition provides nutrients through a nasogastric tube, and parenteral nutrition is injected intravenously. Fortified nutritional preparations provide energy for the intestinal mucosa and not only maintain its barrier function but also prevent intestinal toxins from entering the bloodstream and causing bacterial translocation $(5,8)$. The condition of patients with TBI is more serious. Nutritional supplements can correct nutritional and metabolic disorders, protect intestinal mucosal function, improve body immunity, promote patient recovery, and reduce postoperative complications $(20,21)$.

Our meta-analysis results showed that the infection rate of the nutritional support group was significantly lower than that of the standard nutrition group, and the difference was statistically significant regardless of the total infection rate or the lung infection rate. The nutritional support treatment group also demonstrated an increased total serum protein level, a shortened ICU stay, and better GCS scores than the standard nutrition group. Traumatic craniocerebral injury is caused by direct or indirect violence to the head, often resulting in extensive skull fractures and brainstem injuries. The treatment of TBI is also one of the most challenging problems for neurosurgery and has a high mortality and disability rate. Intensified enteral nutrition can effectively improve the immune function of patients with TBI, thereby reducing the infection rate and promoting recovery. Cooperating with scientific and reasonable nursing plans is also an essential means of ensuring the smooth implementation of enteral nutrition $(18,22,24)$.

In this study, only Chinese and English-language articles were searched. Inevitably, this strategy resulted in an incomplete article collection, which may have created a reduced quality and weaker trends in the research results. A total of seven articles and 512 study subjects were included in this meta-analysis. Because the sample size in the included studies was relatively small, and the articles included in this study were heterogeneous in sample and methodology, the specifics of the nutritional plan could not be studied. In addition, because positive results are easier to publish than negative results there is always some inherent publication bias, which may affect the authenticity, validity, and safety of any conclusions.

\section{Conclusions}

In summary, the effective implementation of nutritional support was shown to increase the total serum protein levels of patients with TBI, reduce the risk of infection, shorten the length of ICU stay, and optimize GCS scores. Although nutritional support does not significantly improve the mortality of patients, it can effectively enhance their nutritional function and immune system. It is recommended for clinical promotion and application. However, due to the limitations of the current research, we recommend that high-quality, large-sample, and multi-center RCTs are conducted in the future to standardize the methods, content, time, and evaluation indicators of nursing implementation of nutritional support to further improve the strength and validity of the current evidence.

\section{Acknowledgments}

Funding: None. 


\section{Footnote}

Reporting Checklist: The authors have completed the PRISMA reporting checklist. Available at https://dx.doi. org/10.21037/apm-21-3071

Conflicts of Interest: All authors have completed the ICMJE uniform disclosure form (available at https://dx.doi. org/10.21037/apm-21-3071). The authors have no conflicts of interest to declare.

Ethical Statement: The authors are accountable for all aspects of the work in ensuring that questions related to the accuracy or integrity of any part of the work are appropriately investigated and resolved.

Open Access Statement: This is an Open Access article distributed in accordance with the Creative Commons Attribution-NonCommercial-NoDerivs 4.0 International License (CC BY-NC-ND 4.0), which permits the noncommercial replication and distribution of the article with the strict proviso that no changes or edits are made and the original work is properly cited (including links to both the formal publication through the relevant DOI and the license). See: https://creativecommons.org/licenses/by-nc-nd/4.0/.

\section{References}

1. Rakhit S, Nordness MF, Lombardo SR, et al. Management and Challenges of Severe Traumatic Brain Injury. Semin Respir Crit Care Med 2021;42:127-44.

2. Takahashi CE, Virmani D, Chung DY, et al. Blunt and Penetrating Severe Traumatic Brain Injury. Neurol Clin 2021;39:443-69.

3. Rai VRH, Phang LF, Sia SF, et al. Effects of immunonutrition on biomarkers in traumatic brain injury patients in Malaysia: a prospective randomized controlled trial. BMC Anesthesiol 2017;17:81.

4. Wijayatilake DS, Shepherd SJ, Sherren PB. Updates in the management of intracranial pressure in traumatic brain injury. Curr Opin Anaesthesiol 2012;25:540-7.

5. Reintam Blaser A, Starkopf J, Alhazzani W, et al. Early enteral nutrition in critically ill patients: ESICM clinical practice guidelines. Intensive Care Med 2017;43:380-98.

6. Schieferdecker ME, Kuretzki CH, Campos AC, et al. New electronic protocol for home enteral nutrition therapy. Arq Bras Cir Dig 2013;26:195-9.

7. Chen L, Liu W, Cheng J. Influence of early enteral nutrition combined with probiotics on postperative infections in patients with traumatic traumatic brain injury. Chin J Nosocomiol 2015;25:1357-9.

8. Tan M, Zhu JC, Yin HH. Enteral nutrition in patients with severe traumatic brain injury: reasons for intolerance and medical management. Br J Neurosurg 2011;25:2-8.

9. Li J, Lu R, Liao T, et al. Application of early enteral nutrition supplemented with probiotics in patients with traumatic craniocerebral injury and its effect on injection and immune function. Chin J Nosocomiol 2018;28:2973-80.

10. Acosta-Escribano J, Fernández-Vivas M, Grau Carmona $T$, et al. Gastric versus transpyloric feeding in severe traumatic brain injury: a prospective, randomized trial. Intensive Care Med 2010;36:1532-9.

11. Dixon J, Comstock G, Whitfield J, et al. Emergency department management of traumatic brain injuries: A resource tiered review. Afr J Emerg Med 2020;10:159-66.

12. Kirby AC, Yrlid U, Svensson M, et al. Differential involvement of dendritic cell subsets during acute Salmonella infection. J Immunol 2001;166:6802-11.

13. Wan G, Wang L, Zhang G, et al. Effects of probiotics combined with early enteral nutrition on endothelin-1 and C-reactive protein levels and prognosis in patients with severe traumatic brain injury. J Int Med Res 2020;48:300060519888112.

14. Tan M, Zhu JC, Du J, et al. Effects of probiotics on serum levels of Th1/Th2 cytokine and clinical outcomes in severe traumatic brain-injured patients: a prospective randomized pilot study. Crit Care 2011;15:R290.

15. Yang $\mathrm{L}, \mathrm{Wu} \mathrm{W}$, Wang $\mathrm{H}$, et al. Influence of probiotics combined with early enteral nutrition on clinical efficacy and intestinal immune level in patients with traumatic craniocerebral injury. Chin J Microecol 2017;29:924-7.

16. Xiong X, Zhou Y, Dong L, et al. Probiotics as supplement for early enteral nutrition decreases infection in traumatic brain injury. J Third Mil Med Univ 2013;35:536-9.

17. Du T, Jing X, Song S, et al. Therapeutic Effect of Enteral Nutrition Supplemented with Probiotics in the Treatment of Severe Craniocerebral Injury: A Systematic Review and Meta-Analysis. World Neurosurg 2020;139:e553-71.

18. Chourdakis M, Kraus MM, Tzellos T, et al. Effect of early compared with delayed enteral nutrition on endocrine function in patients with traumatic brain injury: an openlabeled randomized trial. JPEN J Parenter Enteral Nutr 2012;36:108-16.

19. Chang AC. Determination of cardiac output in critically ill children: are we any closer to the ideal methodology? 
Pediatr Crit Care Med 2012;13:99.

20. Chiang YH, Chao DP, Chu SF, et al. Early enteral nutrition and clinical outcomes of severe traumatic brain injury patients in acute stage: a multi-center cohort study. J Neurotrauma 2012;29:75-80.

21. Kurtz P, Rocha EEM. Nutrition Therapy, Glucose Control, and Brain Metabolism in Traumatic Brain Injury: A Multimodal Monitoring Approach. Front Neurosci 2020;14:190

22. Li X, Yang Y, Ma ZF, et al. Enteral combined with parenteral nutrition improves clinical outcomes in patients with traumatic brain injury. Nutr Neurosci 2020. [Epub

Cite this article as: Yang L, Liao D, Hou X, Wang Y, Yang C. Systematic review and meta-analysis of the effect of nutritional support on the clinical outcome of patients with traumatic brain injury. Ann Palliat Med 2021;10(11):11960-11969. doi: 10.21037/apm-21-3071 ahead of print].

23. Yi LJ, Tian X, Shi B, et al. Early enteral nutrition supplemented with probiotics improved the clinical outcomes in severe head injury: Some promising findings from Chinese patients. Medicine (Baltimore) 2019;98:e15426.

24. Vella MA, Crandall ML, Patel MB. Acute Management of Traumatic Brain Injury. Surg Clin North Am 2017;97:1015-30.

(English Language Editor: D. Fitzgerald) 